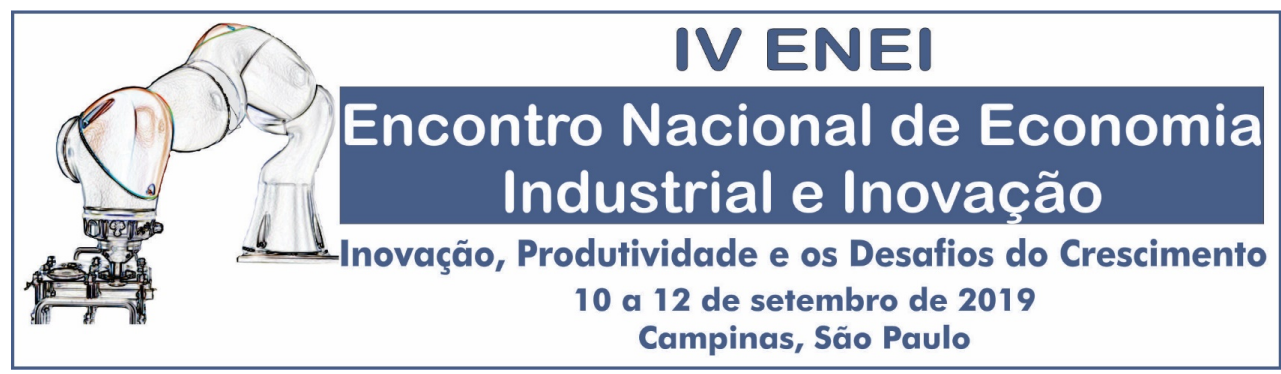

\title{
POLÍTICAS INDUSTRIAIS NO CONTEXTO DO COMPLEXO ECONÔMICO-INDUSTRIAL DA SAÚDE: o caso das Parcerias para o Desenvolvimento Produtivo
}

Gabriela Rocha Rodrigues de Oliveira ${ }^{1}$ Ana Lucia Gonçalves da Silva ${ }^{2}$

\section{RESUMO:}

O presente trabalho, partindo do arcabouço teórico consubstanciado pelo conceito Complexo EconômicoIndustrial da Saúde, procura realizar um levantamento das políticas industriais realizadas na indústria de saúde brasileira, entre os anos de 1998 e 2014, com ênfase nas Parcerias para o Desenvolvimento Produtivo (PDPs), como símbolo de um esforço sistêmico visando à conciliação de políticas que proporcionem a capacitação tecnológica do parque produtivo brasileiro e o fortalecimento do Sistema Único de Saúde (SUS). Dentro da análise, procurou-se evidenciar a arquitetura proposta pela política, seus principais elementos e uma breve avaliação de seus impactos e resultados preliminares. Conclui-se que, apesar de ainda muito elevadas as dificuldades associadas à indústria de saúde brasileira, as iniciativas que se propõem a enfrentar tais desafios, como o caso das PDPs, tornam-se cada vez mais imperativas diante de um cenário em que a sustentação material do SUS tem sido colocada sob crescente risco.

Palavras-Chave: Parcerias para o Desenvolvimento Produtivo; Complexo Econômico-Industrial da Saúde; Politicas Industriais; Sistema Único de Saúde.

\section{ABSTRACT:}

The present work, based on the theoretical framework incorporated by the Economic-Industrial Health Complex concept, aimed at carrying out a survey of the implemented industrial policies in the Brazilian health industry between 1998 and 2014, with emphasis on Productive Development Partnerships, as symbol of a systemic effort aimed at conciliating policies that provides technological capacity to the brazilian industry and, at the same time, strengthens of the Unified Health System (SUS). The analysis highlights the architecture proposed by the policy, its main elements and makes a brief evaluation of its preliminary impacts and results. It is concluded that, although the difficulties associated with the Brazilian health industry are still very high, the initiatives that propose to face such challenges become increasily imperative in the face of a scenario in which the material conditions to SUS sustentation has been placed under increasing risk.

Key-words: Productive Development Partnerships; Economic-Industrial Health Complex; Industrial Policies; Unified Health System (SUS).

ÁREA (ABEIN): Políticas Públicas e Regulação: Políticas de Ciência, Tecnologia e Inovação;

CLASSIFICAÇÃO JOURNAL ECONOMIC LITERATURE (JEL): O25 (Economic Development, Innovation, Technological Change, and Growth: Development Planning and Policy: Industrial Policy).

\footnotetext{
${ }^{1}$ Economista, mestranda em Desenvolvimento Econômico, com ênfase em Economia Social e do Trabalho, pela Universidade Estadual de Campinas (UNICAMP). E-mail: gabrielarrdeoliveira@gmail.com.

${ }^{2}$ Economista, doutora em Ciências Econômicas pela Universidade Estadual de Campinas (Unicamp). Professora do Instituto de Economia (IE) da Unicamp. Professora do Programa de Pós-Graduação em Desenvolvimento Econômico do IE/Unicamp. Membro do Conselho Superior do Núcleo de Estudos de Políticas Públicas (NEPP) da Unicamp. E-mail: algsilva@unicamp.br.
} 


\section{INTRODUÇÃO}

\subsection{O COMPLEXO ECONÔMICO-INDUSTRIAL DA SAÚDE}

O conceito de Complexo Econômico-Industrial da Saúde (CEIS, o qual era denominado, inicialmente, como Complexo Industrial da Saúde) foi introduzido por Gadelha (2003), como uma forma de viabilizar a conciliação das perspectivas de desenvolvimento da estrutura produtiva brasileira com desenvolvimento social, associado, nessa perspectiva, à consolidação e ao fortalecimento do Sistema Único de Saúde (SUS). Para isso, o autor analisa a saúde por meio de uma abordagem sistêmica: a oferta de serviços de saúde não estaria desvinculada, dentro dessa visão, a todos os setores produtores dos bens necessários à oferta desses serviços. Partindo-se dessa perspectiva, portanto, a defesa do direito à saúde, diretamente atrelada ao fortalecimento do SUS, ultrapassaria a sua já incontestável importância civilizatória, principalmente diante do tamanho dos abismos sociais enfrentados pelo Brasil, e passaria a apresentar também uma grande oportunidade de dinamização da economia, se esforços adequados fossem realizados nesta direção (GADELHA, 2017).

$\mathrm{O}$ argumento fica mais claro ao serem analisados alguns números relacionados à saúde no Brasil: de acordo com Gadelha (2017), se pensada como sistema integrado e articulado, para além de uma análise fragmentada dos segmentos de medicamentos e vacina, a saúde no Brasil mobiliza o equivalente a 10\% do PIB, o que representaria recursos na faixa de $\mathrm{R} \$ 600$ bilhões na economia brasileira. Também à saúde estão vinculados 12 milhões de trabalhadores qualificados e 35\% das pesquisas realizadas no Brasil (incluindo as áreas de ciências biológicas e biomédicas) (GADELHA, 2017). É evidente, portanto, que a questão da garantia do direito à saúde no Brasil não pode ser analisada apenas por meio da ótica da oneração fiscal: muitas outras possibilidades são abertas quando se evidencia a saúde por meio de uma perspectiva sistêmica e o argumento de que um sistema público, universal e gratuito seria incompatível com as capacidades fiscais do Estado brasileiro poderia ser, em grande medida, relativizado (GADELHA; TEMPORÃO, 2018).

Esse argumento é reforçado quando são analisados os efeitos multiplicadores de renda dos gastos em saúde: um estudo realizado por Abrahão, Mostafa e Herculano (2011) estimou que, a cada R $\$ 1,00$ gasto em saúde, há um retorno ao PIB de $\mathrm{R} \$ 1,70$. Na renda das famílias o retorno é menor, mas ainda assim relevante, uma vez que o efeito-multiplicador estimado é de 1,44. O mesmo estudo demonstrou que, por meio do crescimento da arrecadação governamental associada à elevação do PIB, estimulada, no modelo proposto, pelo aumento dos gastos com políticas sociais e os seus respectivos efeitos multiplicadores, haveria um retorno estimado do equivalente a $56 \%$ dos gastos aos cofres públicos, o que significaria que parte do dispêndio governamental com políticas sociais seria revertido ao Estado brasileiro após o ciclo de multiplicação de renda por ele engendrado (ABRAHÃO; MOSTAFA; HERCULANO, 2011). Os efeitos multiplicadores dos gastos sociais, de acordo com Orair, Siqueira e Gobetti (2016), tornam-se ainda mais relevantes quando a economia encontra-se em fase recessiva do ciclo econômico.

No entanto, apesar da grande relevância do CEIS para a economia brasileira, muitos desafios apresentam-se tanto nos âmbitos da oferta de serviços de saúde, quanto da indústria associada à produção dos bens utilizados para que tais serviços possam ser oferecidos. O primeiro desafio, apontado por muitos autores como uma das principais barreiras ao fortalecimento do SUS, é o subfinanciamento da saúde pública no Brasil (SALDIVA; VERAS, 2018). Desde o seu surgimento, em 1988, o SUS passa por grandes dificuldades relacionadas à obtenção de um financiamento adequado às dimensões da proposta constitucional que carrega: consolidar-se como um sistema público, único, universal e gratuito, garantindo o acesso à saúde a todos os cidadãos brasileiros. Isso é especialmente relevante quando analisado sob a perspectiva de que o Estado brasileiro, após a criação do SUS, passava por importantes mudanças de paradigma, aderindo de forma ampla a políticas neoliberais, as quais procuravam reduzir e forma sistemática a participação do Estado na coordenação da economia e privilegiando políticas sociais 
focalizadas, em detrimento de uma perspectiva universalista, que regeu, em grande medida, a construção da Constituição Federal de 1988 (FAGNANI, 2005). Por mais que o SUS configure-se entre os maiores sistemas de saúde pública do mundo, o orçamento destinado ao seu financiamento equivaleu, em 2015, a US\$ 595,00 per capita, o que representa o dispêndio médio inferior a países latino-americanos e muito aquém aos gastos realizados em países possuidores de sistemas universais de saúde, como a França e a Inglaterra (SALDIVA; VERAS, 2018).

Em relação ao gasto realizado com saúde baseado no tamanho do PIB brasileiro, o que se observa é que, apesar do gasto total apresentar valores equivalentes a, em média, 10\% do PIB, em 2006, 66\% estão associados a gastos privados e $44 \%$ a gastos públicos. Países da OCDE, neste mesmo ano, possuíam, uma média de proporção de gastos públicos em saúde de 73,1\%. Os países do Mercosul, por sua vez, desconsiderando-se o Brasil, tinham a média de gastos públicos da ordem de 51,2\% dos gastos totais (GADELHA et al., 2010). Isso diante de uma situação em que, em 2015, 72\% da população depende exclusivamente do SUS (CONSELHO FEDERAL DE MEDICINA, 2015), gera um contexto em que o gasto per capita privado em saúde ultrapassa em larga escala os gastos destinados ao SUS. Tal situação, somada às perspectivas de envelhecimento e da mudança do perfil epidemiológico da população e das limitações orçamentárias crescentes, dadas, por exemplo, pela Emenda Constitucional 95, tornam as perspectivas da sustentação e aprimoramento do SUS questões cada vez mais urgentes e necessárias de serem aprofundadas no debate nacional (OCKÉ-REIS; FUNCIA, 2016).

Um segundo desafio deve ser apontado. Para além das dificuldades no âmbito do financiamento do SUS, o país também apresenta um cenário de crescente fragilidade produtiva na indústria da saúde, principalmente por parte dos setores de maior densidade tecnológica e mais intensivos em inovação. A Figura 1, a seguir, demonstra a trajetória crescente do déficit comercial associado aos setores industriais ligados à saúde no Brasil, entre os anos de 1996 e 2015.

Figura 1: Balança comercial da saúde: panorama geral, 1996-2015 (em bilhões de US\$)

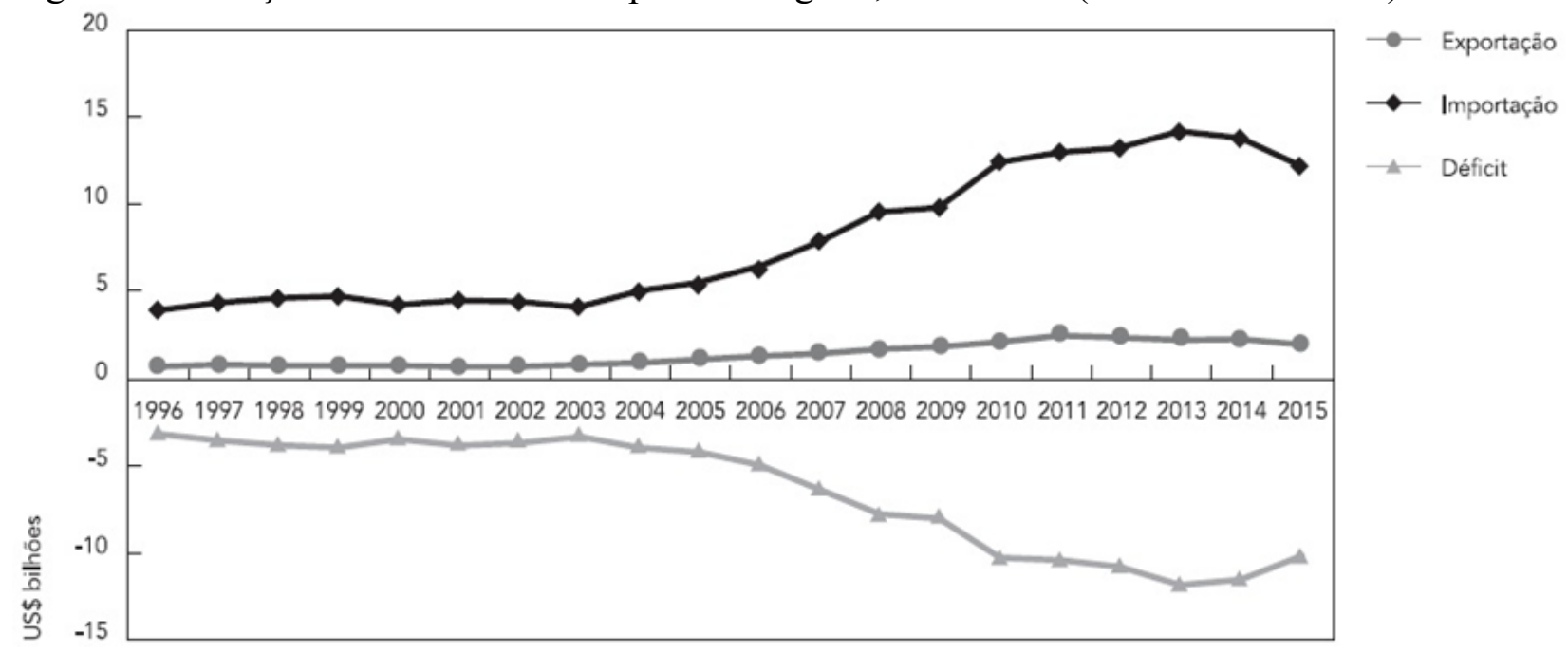

Fonte: GADELHA; BRAGA, 2016, p. 9.

Dentro deste panorama, há uma tendência de elevação da participação de produtos de maior intensidade tecnológica na composição do déficit, demonstrando um cenário de fragilidade tecnológica crescente do parque produtivo brasileiro ligado à saúde, principalmente diante de um movimento da indústria mundial em que a saúde tem vivenciado mudanças radicais de paradigma, crescentemente caminhando no sentido de promover inovações de grande estatura nos tratamentos e nos recursos necessários à realização deles (PADULA; NORONHA; MITIDIERI, 2016). Esse aspecto pode ser representado pelo movimento da balança comercial relacionada aos medicamentos biotecnológicos, demonstrada pela Figura 2. 
Figura 2: Balança comercial do setor de produtos farmacêuticos biotecnológicos, 2000-2015 (em bilhões de US\$)

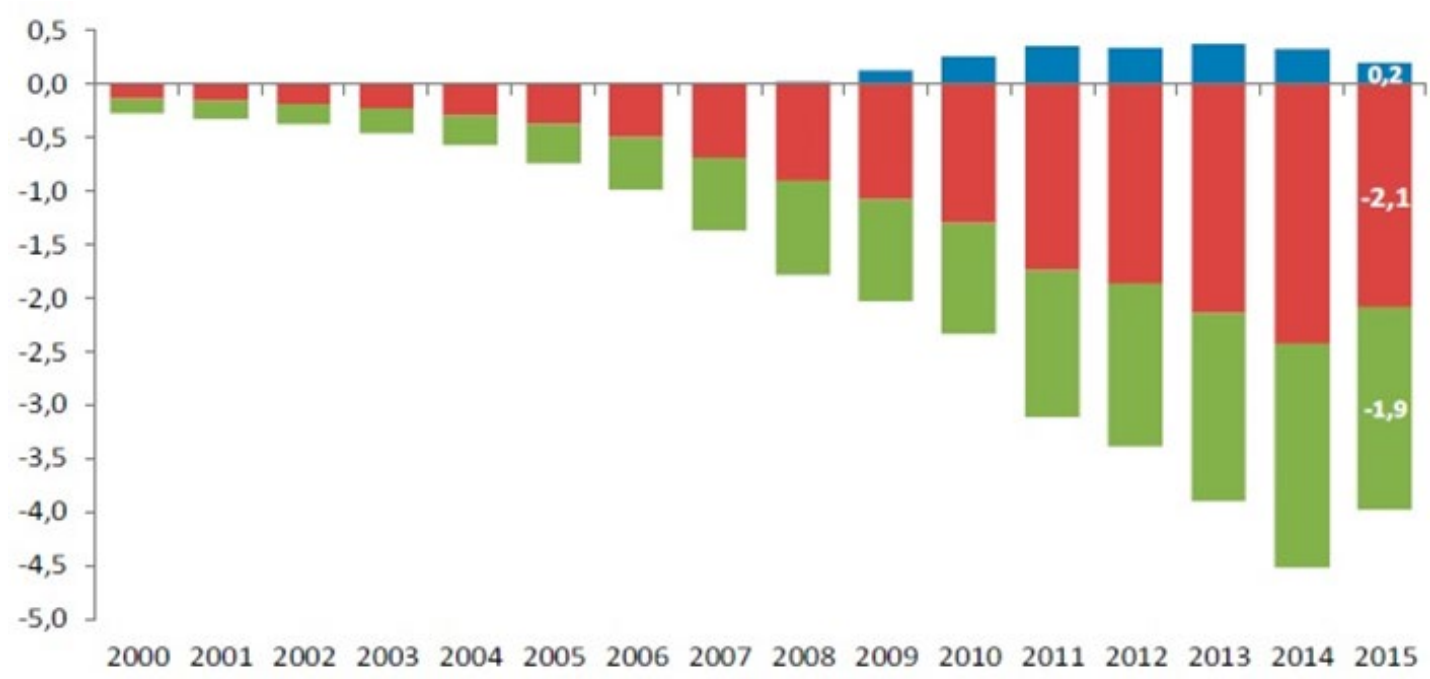

Exportação $\quad$ Importação Saldo Comercial

Fonte: NOVAIS; QUINTÃO; CAGNIN, 2016, p. 28.

Diante do breve panorama apresentado, fica evidente que a questão da saúde no Brasil, em especial associada à sustentabilidade e ao fortalecimento do SUS, apesar dos grandes avanços realizados pelo sistema nos trinta anos de sua existência, sendo a única porta de acesso à saúde de grande parte da população, enfrenta grandes fragilidades, que tendem a se agravar caso não haja um enfrentamento adequado aos desafios crescentes que vêm sendo impostos. A perspectiva do Complexo EconômicoIndustrial da Saúde caminha em direção à superação das duas principais fontes de fragilidades apresentadas. Por um lado, ao enxergar a saúde por uma ótica sistêmica, compreende os grandes desafios existentes para o desenvolvimento da indústria brasileira ligada à saúde, principalmente em relação aos setores mais intensivos em tecnologia, colocando o país em uma situação de crescente vulnerabilidade, reduzindo suas possibilidades de desenvolvimento de um sistema de saúde público e universal (GADELHA; COSTA, 2013).

Logo, uma estratégia de longo prazo de política industrial por parte do Estado brasileiro, que buscasse concentrar esforços para enfrentar as dificuldades estruturais dos setores industriais ligados à saúde no país, poderia ser um caminho para a diminuição da vulnerabilidade do país frente aos movimentos da indústria mundial (e, no âmbito geopolítico, das potências a ela associadas, uma vez que, como expõem Padula, Noronha e Mitidieri (2016), elas possuem estratégias de segurança nacional claras direcionadas aos setores intensivos em tecnologia ligados à saúde, proporcionando expressivos investimentos em seus desenvolvimentos). Como estes setores possuem características de elevados gastos em inovação, este poderia ser um importante passo em direção à transformação da estrutura produtiva do país, capacitando a indústria brasileira em tecnologias-chave, que poderiam penetrar diversos setores produtivos, agregando valor à produção nacional (GADELHA; COSTA, 2013).

Por outro lado, a perspectiva do CEIS também abre espaço para a superação dos desafios ligados ao financiamento do SUS: as estratégias de política industrial, a partir desta ideia, não estariam dissociadas das estratégias de política social, muito pelo contrário. O fomento do desenvolvimento produtivo por parte do Estado estaria diretamente relacionado à perspectiva de fortalecimento SUS, uma vez que deveria ser coordenada de forma a atender as necessidades apresentadas pelo sistema. A política industrial estaria, portanto, imersa em uma lógica de fornecimento dos bens necessários à oferta de saúde à população por meio do SUS. Dentre os muitos mecanismos de coordenação necessários à concretização deste arranjo, Gadelha e Temporão (2018) destacam as compras públicas como elementos fundamentais (já que seriam capazes de relacionar de forma direta as esferas produtiva e de oferta de serviços), bem como a existência (e o fortalecimento) dos laboratórios farmacêuticos oficiais (LFOs), que poderiam ser 
elementos de elevação da autonomia do Estado para o acesso a bens de maior valor agregado, representando uma possibilidade de maior controle sanitário e de variação de preços.

Mesmo diante dos grandes desafios históricos e conjunturais associados à questão da saúde no Brasil, o período recente, ainda que distante de consolidar um padrão de saúde pública universal e de qualidade no Brasil, demonstrou iniciativas importantes em direção a uma leitura sistêmica da saúde no país, em que diversas instituições reuniram-se em torno da temática e fizeram ensaios em direção a políticas industriais que convergissem em direção à sustentação e ao fortalecimento do SUS. Tais políticas serão o objeto de estudo deste artigo, com especial destaque às Parcerias para o Desenvolvimento Produtivo (PDPs), que representaram, de acordo com Gadelha e Temporão (2018), a forma mais aprimorada até o momento da conciliação de políticas que, simultaneamente, visassem à capacitação tecnológica do parque produtivo brasileiro e ao desenvolvimento do SUS. A próxima seção fará uma retrospectiva breve sobre como estiveram inseridos os setores industriais relacionados à saúde nas grandes frentes de política industrial lançadas entre 2004 e 2011, enfatizando, no segundo item, o caso das Parcerias para Desenvolvimento Produtivo. Por fim, na última seção, serão apresentadas as considerações finais, nas quais serão feitas reflexões sobre como a temática articula-se com os encaminhamentos recentes políticos e econômicos no Brasil, sem que, no entanto, intencione-se esgotar a discussão do tema - ainda passível de ricas possibilidades de estudo e aprofundamentos.

\section{POLÍTICAS INDUSTRIAIS NO CONTEXTO DO CEIS}

Diante da ausência de políticas industriais ativas na década 1990, o início dos anos 2000, com a entrada do presidente Luiz Inácio Lula da Silva, em 2003, representou uma importante mudança paradigmática, trazendo de volta a temática ao debate nacional (CANO; SILVA, 2010). Entre 2004 e 2011, foram lançadas três diretrizes de política industrial, que buscaram, dentro dos contextos políticos e econômicos em que se inseriram, responder às dificuldades vivenciadas pela indústria brasileira.

Os setores relacionados à saúde estiveram entre as diretrizes desde a primeira versão da política industrial, lançada em 2004, e foram objetos de preocupação crescente por parte do Governo, pela relevância que apresentam, não só em termos do considerável potencial de inovação e geração de valor agregado que carregam, mas também pela grande importância social (OLIVEIRA, 2017). A forma como esses setores inseriram-se dentro das grandes diretrizes de política industrial realizadas nas últimas duas décadas será o objeto a ser tratado pela próxima seção.

\subsection{A saúde nas "grandes frentes" de políticas industriais}

A década de 1990 trouxe grandes desafios relacionados à questão da saúde no Brasil: ao mesmo tempo em que a saúde, por determinação constitucional, tornou-se um direito da população, o que implicaria uma expansão do dispêndio relacionado a tal garantia, a estrutura industrial brasileira passava por uma reestruturação em direção a setores menos intensivos em tecnologia, perdendo espaço dentro do valor agregado produzido no país (CALDEIRA, 2010). Isso afetou de forma direta os setores produtores de bens ligados à saúde, uma vez que diversos ramos da "indústria da saúde", tais como os pertencentes aos subsistemas de base química e biotecnológica e de base mecânica, eletrônica e de materiais, são intensivos em inovação e fazem parte das fronteiras tecnológicas mundiais (GADELHA; COSTA; MALDONADO, 2012). Dentro desse panorama, mesmo diante de uma diretriz política geral de ausência de políticas industriais no país, ainda em 1998, pela Portaria 3.196, foi lançada a Política Nacional de Medicamentos (PNM), que, apesar de não ser caracterizada como uma política industrial, tinha, entre os seus objetivos, enfrentar a grande perda de dinamismo que a indústria farmacêutica brasileira vinha 
passando durante a década de 1990. Suas diretrizes relativas à indústria farmacêutica nacional eram, principalmente, a regulamentação sanitária dos medicamentos, o desenvolvimento científico e tecnológico desta indústria, a promoção da produção de medicamentos em solo nacional e a garantia de padrões de segurança, eficiência e qualidade dos produtos (BRASIL, 2001).

Essa lei abriu espaço para a promulgação de duas importantes leis: a Lei 9.787, de 1999, conhecida como Lei dos Genéricos, a qual viria a promover uma relevante reestruturação na produção nacional de medicamentos, pois ampliou as possibilidades de mercado para as empresas de capital nacional, além de reduzir, de forma considerável, os preços dos medicamentos de patentes vencidas para as compras governamentais e da população (GOMES et al., 2013). Além disso, a partir da PNM, foram dadas as diretrizes para a criação da Agência Nacional de Vigilância Sanitária (ANVISA), por meio da Lei 9.782, de 1999. Este tem sido um órgão importante para a regularização e padronização da produção de medicamentos no país (inclusive os genéricos) e das vendas à população (BRASIL, 2001; SILVA, 2014). Esses movimentos, ainda que limitados, tanto no âmbito do número de setores envolvidos, quanto pelo baixo dinamismo inovativo e tecnológico associado à indústria de medicamentos genéricos, foram importantes para criar uma base mínima para a atuação das políticas industriais que se fortaleceriam no início dos anos 2000 (CAPANEMA, 2006).

Como exposto no item anterior, a mudança governamental em 2003 representou um retorno da busca pela atuação direta do Estado brasileiro para o fomento da indústria nacional (CANO; SILVA, 2010). Entre 2004 e 2011, foram lançadas três grandes frentes de política industrial: a primeira delas, lançada em 2004, foi a Política Industrial, Tecnológica e de Comércio Exterior (PITCE). Em 2008, foi lançada a Política para o Desenvolvimento Produtivo (PDP) e, em 2011, foi lançado o Plano Brasil Maior (PBM). É importante ressaltar que a ausência de políticas industriais durante a década de 1990, como expõem Cano e Silva (2010), trouxe grandes dificuldades institucionais (pois os instrumentos de política tiveram que, em grande medida, ser reconstruídos em bases completamente novas) e também relacionadas à concepção adequada de mecanismos que fossem capazes de fazer frente aos movimentos da globalização produtiva e financeira, em acelerado processo. Outro ponto considerado de extrema relevância para os autores refere-se à questão de que houve uma grande contradição entre a realização, de um lado, de políticas industriais, que buscavam elevar o investimento industrial no Brasil, e de outro as diretrizes da política macroeconômica, que se mantinham alinhadas aos preceitos neoliberais, baseada no "tripé macroeconômico" (metas de inflação, câmbio flutuante e superávit primário) e em elevada taxa básica de juros para que tais metas pudessem ser cumpridas, bloqueando maiores possibilidades de transformação da estrutura produtiva brasileira. Além disso, em 2008, o mundo passa por uma crise econômica de dimensões muito elevadas, que muda de forma radical os cenários da economia brasileira e mundial, que necessitaram se adaptar às necessidades conjunturais impostas. Portanto, a avaliação objetiva geral sobre a eficiência e a eficácia dessas políticas não constitui tarefa simples e não faz parte do escopo a ser abordado pelo ensaio. No entanto, nos setores ligados à saúde, iniciativas importantes foram tomadas, de forma que se incorporou, de maneira progressiva, às diretrizes governamentais, a análise da saúde por meio de uma perspectiva sistêmica. Tal movimento será expresso nos parágrafos seguintes.

Para Gadelha e Costa (2013), a PITCE, lançada em 2004, pode ser considerada um marco na institucionalização do caráter estratégico da produção para a área da saúde nas políticas governamentais. Ela, conforme exposto por Cano e Silva (2010), englobou tanto linhas de ação horizontais ("inovação e desenvolvimento tecnológico; inserção externa; modernização industrial; ambiente; institucional/aumento da capacidade produtiva (p.7)"), quanto opções estratégicas ("semicondutores, software, bens de capital e fármacos (p.7)") e atividades portadoras de futuro ("biotecnologia, nanotecnologia, biomassa/energias renováveis (p.7)"). A política colocou entre as suas grandes prioridades a busca pela inovação do setor farmacêutico, definindo-o como um setor tanto estratégico, quanto articulado a tecnologias do futuro, tais como nanotecnologia, química fina e biotecnologia (GADELHA; COSTA, 2013; SILVA, 2014). Ela foi importante para o desenvolvimento de diversas ações, tais como o programa do Banco Nacional de Desenvolvimento (BNDES) de Apoio ao Desenvolvimento da Cadeia Produtiva Farmacêutica (PROFARMA), a Lei da Inovação, a Lei do Bem. Em 2004, foi lançada a Política Nacional de Ciência, Tecnologia e Inovação em Saúde, que refletia a preocupação governamental com o desenvolvimento de 
maior autonomia industrial no Brasil, mas que foi concretizar-se de forma mais efetiva em 2007, com a inclusão do Complexo Industrial da Saúde (CIS) como estratégia em destaque no programa Mais Saúde (GADELHA; COSTA, 2013; OLIVEIRA, 2017).

Em 2008, em busca da superação de lacunas deixadas pela PITCE e para a continuidade do enfrentamento dos desafios colocados à estrutura industrial brasileira, implementou-se a Política de Desenvolvimento Produtivo (PDP). Dentre os diversos setores beneficiados (sendo eles 25, no total), o Complexo Industrial da Saúde (CIS) estava dentro dos setores estratégicos devido ao seu grande potencial de disseminação de conhecimento e inovação (SILVA, 2014) e as ações envolvidas para o seu fortalecimento teriam entre as diretrizes o incentivo à produção e à inovação em saúde no Brasil, com objetivos de elevação da competitividade em âmbitos interno e externo, a garantia da isonomia na regulação sanitária e de medidas de apoio à qualidade da produção brasileira, o estímulo ao uso do poder de compra do Sistema Único de Saúde para favorecer a produção, competitividade e inovação do CIS e a criação de rede de suporte à qualidade e à competitividade da produção local.

De acordo com Gadelha e Temporão (2018), a PDP foi um importante marco para a leitura da saúde a partir de uma perspectiva sistêmica. Concomitante ao seu lançamento, houve a criação do Grupo Executivo para o Complexo Industrial da Saúde (GECIS), que teve o papel de instância política de coordenação, envolvendo a representação formal de catorze órgãos do Estado, estando entre eles a Casa Civil, os Ministérios da Fazenda, do Planejamento, das Relações Exteriores, do Desenvolvimento, Indústria e Comércio e da Ciência Tecnologia e Inovação, além de agências nacionais de fomento e instituições de ciência, tecnologia e inovação. Todas as ações do grupo seriam voltadas ao atendimento das necessidades apresentadas pelo SUS.

Também na PDP desenvolveram-se instrumentos de uso do poder de compra do Estado atrelado ao SUS, que se constituiu como importante mecanismo de indução e de regulação da produção nacional. Como expõem Gadelha e Temporão (2018):

De um lado, o poder de compra e as políticas estatais orientadas por missão constituem a principal força por trás de processos mais substantivos e arriscados de transformação produtiva. De outro lado, o papel estratégico desse instrumento de intervenção pública se relaciona ao próprio vínculo defendido entre as dimensões econômicas e as sociais do desenvolvimento. O elo crítico dessa relação, e que subordina a política industrial e de inovação às necessidades do sistema de saúde, está justamente no atendimento às demandas do SUS (p. 1896).

Em 2011, foi lançado o Plano Brasil Maior (PBM), que procurou dar continuidade à PITCE e à PDP, dando as diretrizes governamentais para as políticas industriais de 2011 a 2014, adaptadas a um contexto econômico adverso da economia mundial. O PBM tinha como meta principal estimular a competitividade, a inovação e o adensamento produtivo da indústria brasileira, por meio de ações tanto estruturantes (voltadas a setores-chave), quanto sistêmicas (voltadas a temas transversais), procurando estimular a reação da indústria aos efeitos que haviam sido causados pela crise econômica mundial que eclodiu em 2008 (BNDES, 2011). O plano reafirmou o protagonismo da saúde como um dos setores que deveriam ser fomentados no Brasil, estabelecendo novas diretrizes para o aumento do uso de poder de compra estatal (ligado ao SUS) (GADELHA; COSTA, 2013). Exemplo deste movimento foi a determinação de "margens de preferência" aos produtos manufaturados nacionais diante das licitações das compras públicas, que atingiram diversos setores estratégicos, entre eles o setor de fármacos e medicamentos (que variavam de 8 a 25\%, conforme o produto determinado) (BRASIL, 2012a), e o de equipamentos médico-hospitalares (que também possuíam variações de 8 a 25\%) (BRASIL, 2012b). Em 2012, foi implantada a Estratégia Nacional de Ciência, Tecnologia e Inovação, a qual procurou enfatizar a importância da busca pela inovação em saúde e pela transferência tecnológica a laboratórios nacionais, tanto públicos, como privados, e as suas conexões com o fortalecimento e aprimoramento do SUS (GADELHA; COSTA, 2013). 
A seção seguinte será dedicada ao maior detalhamento das Parcerias para o desenvolvimento produtivo (PDPs), que têm início em 2009 e fortalecem-se em termos de escala e marcos regulatórios até 2014 (GADELHA; TEMPORÃO, 2018).

\subsection{O caso das Parcerias para o Desenvolvimento Produtivo (PDPs)}

As Parcerias para o Desenvolvimento Produtivo (PDPs), coordenadas pelo Ministério da Saúde (MS), foram instituídas em 2008, no mesmo ato de lançamento da PDP, e tiveram início entre os anos de 2009 e 2010, com aprimoramento de seu marco regulatório em 2014. Seu objetivo principal é o de tornar concreta a perspectiva de análise sistêmica da saúde, fomentando a coordenação entre políticas tecnológicas e produtivas, procurando-se a ampliação da autonomia nas áreas mais críticas de dependência tecnológica, e as necessidades apresentadas pelo SUS, por meio de publicações de listagens com produtos estratégicos ao sistema (GADELHA; TEMPORÃO, 2018).

Para tal concretização, três elementos tornam-se essenciais: o primeiro deles são as empresas portadoras das tecnologias consideradas estratégicas pelo MS. O segundo seriam os Laboratórios Farmacêuticos Oficiais (LFOs) e o terceiro o uso do poder de compra do MS. A articulação mais geral entre esses três elementos funciona da seguinte forma: anualmente, o Ministério da Saúde lança uma lista com produtos estratégicos ao SUS. A escolha destes produtos é priorizada conforme os critérios de custo, complexidade tecnológica, participação de importações nas compras totais e escassez de abastecimento (produtos negligenciados). Para a realização das PDPs, as instituições públicas e privadas envolvidas devem desenvolver e enviar um projeto, que será ou não aprovado pela Secretaria de Ciência, Tecnologia e Insumos Estratégicos (SCTIE) do MS. As instituições privadas capacitadas tecnologicamente para desenvolver os produtos devem comprometer-se com as normas sanitárias estipuladas e com a capacitação tecnológica e produtiva de laboratórios oficiais que estejam aptos para tal (sendo, de acordo com Gadelha e Temporão (2018), os principais a Fiocruz e o Instituto Butantan). Isso garante às empresas privadas a exclusividade de compras governamentais durante o período que elas possuem para realizarem a transferência completa da capacitação tecnológica e produtiva dos laboratórios públicos parceiros. O prazo das PDPs varia conforme o grau avaliado de complexidade tecnológica envolvida na produção, podendo alcançar até dez anos para os produtos mais complexos e estratégicos (BRASIL, 2014). Após este prazo, intenciona-se que estes estejam aptos a produzir, de forma autônoma, os produtos estipulados, sempre obedecendo aos princípios de "economicidade e vantajosidade" (GADELHA; TEMPORÃO, 2018, p. 1897). As fases do processo de realização das PDPs estão explicitadas na Figura 3, a seguir.

Figura 3: Etapas de uma Parceria para o Desenvolvimento Produtivo

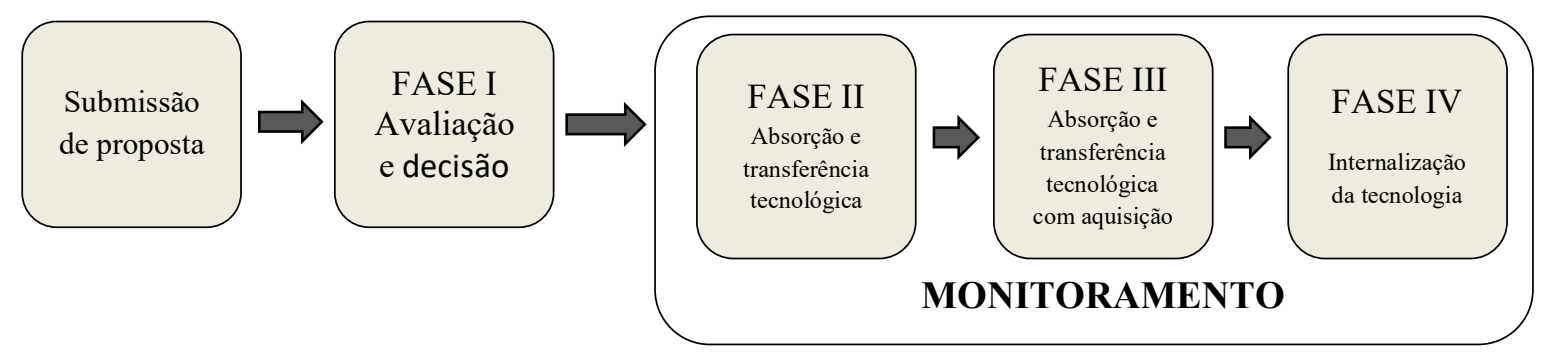

Fonte: BRASIL, 2019.

A fase de submissão de proposta deve ser feita pela instituição pública interessada, dentro do período estipulado pelo Ministério da Saúde, respeitando a listagem dos produtos considerados elegíveis, previamente divulgados. A fase de avaliação e decisão consiste, como o próprio nome indica, na avaliação da viabilidade da proposta submetida, a ser realizada pela Comissão Técnica de Avaliação (CTA), coordenada pela Secretaria de Ciência, Tecnologia e Insumos Estratégicos (SCTIE) do MS. O 
parecer da CTA é analisado pelo Comitê Deliberativo e este aprova ou não a PDP. No caso de aprovação, avança-se para a segunda fase, na qual é iniciada a implementação da PDP, do termo de compromisso, bem como o monitoramento. Há nesta fase o co-desenvolvimento do produto (envolvendo as instituições privada e pública) ou a entrega do dossiê de registro da entidade privada à instituição pública. Também nesta fase há a capacitação industrial e tecnológica para a produção e o registro sanitário do produto, a ser concedido pela ANVISA. A terceira fase representa o início da execução do desenvolvimento do produto e a efetivação da transferência e absorção da tecnologia pela instituição pública. Além disso, há a celebração do contrato de aquisição do produto entre esta e o MS. Ao final desta fase, o Laboratório Farmacêutico Oficial (LFO) deve ser capaz de realizar a produção do item acordado pela PDP em sua própria planta fabril, realizando todos os procedimentos necessários à submissão do registro sanitário do produto proveniente da instituição pública. Por fim, a fase IV é caracterizada pela concretização da internalização da tecnologia, em condições de portabilidade tecnológica entre a instituição pública e a entidade parceira privada, demarcando a possibilidade de desvinculação do registro matriz (proveniente da instituição privada), caracterizando a finalização da PDP. É importante ressaltar que o monitoramento, conforme exposto pela Figura 3, acima, ocorre de forma contínua, entre as fases II e IV. Tal procedimento envolve a fiscalização do cumprimento do cronograma acordado, bem como das responsabilidades assumidas por todas as instituições envolvidas. Há também o monitoramento referente à regulação sanitária, a ser realizado pela ANVISA, e do processo técnico de transferência e de absorção de tecnologia e do desenvolvimento das capacidades da instituição pública para o novo patamar tecnológico, que fica a cargo da SCTIE (BRASIL, 2019).

A coordenação do processo envolvendo as PDPs é feita pelo GECIS (Grupo Executivo do Complexo Industrial da Saúde), que é composto, segundo a Política Nacional de Inovação Tecnológica na Saúde, instituída pelo Decreto 9.307, de 21/12/2017, pelos seguintes órgãos: o Ministério da Saúde (coordenador), a Casa Civil, os Ministérios das Relações Exteriores, da Fazenda, da Educação, da Indústria, Comércio Exterior e Serviços, do Planejamento, Desenvolvimento e Gestão e o da Ciência, Tecnologia, Inovações e Comunicações, a ANVISA, a Fundação Oswaldo Cruz (Fiocruz), o Banco Nacional de Desenvolvimento Econômico e Social (BNDES), o Instituto Nacional de Propriedade Industrial (INPI), a Agência Brasileira de Desenvolvimento Industrial (ABDI), o Instituto Nacional de Metrologia, Qualidade e Tecnologia (INMETRO) e, finalmente, a Financiadora de Estudos e Projetos (FINEP). Constam como objetivos a serem perseguidos pelo grupo a promoção da articulação entre os órgãos públicos e privados envolvidos na produção e prestação de serviços de saúde, bem como o fomento de um ambiente propício ao desenvolvimento industrial e tecnológico do CEIS, em que prevaleçam condições de segurança jurídica e institucional e em que se amplie o acesso a produtos e serviços estratégicos para o fortalecimento do SUS (BRASIL, 2017).

Como expõem Gadelha e Temporão (2018), o número de órgãos representantes na composição do GECIS (sendo 15 ao total) reflete um amplo esforço em direção a uma abordagem sistêmica da saúde, em que se procurou de forma sistemática a convergência entre as distintas áreas do governo e os seus eixos de intervenção, envolvendo instituições com poder de compra, financiamento, ciência e tecnologia, regulação, relações exteriores, entre outras. Esta política também refletiu um esforço de uso do poder do compra do Estado brasileiro como um instrumento de desenvolvimento de via dupla, em que podem ser contempladas, simultaneamente, as suas dimensões sociais e produtivas, rumo a um SUS com maiores possibilidade de universalização de uma saúde pública de qualidade no Brasil, com maior autonomia tecnológica e produtiva, reduzindo a sua vulnerabilidade e estimulando a geração de investimentos, empregos e renda no país.

A avaliação das PDPs, em termos do impacto sobre a estrutura produtiva brasileira, necessita, de acordo com Gadelha e Temporão (2018), de maior tempo para poder ser feita de maneira mais precisa. No entanto, alguns resultados expressivos já podem ser observados em termos do número de parcerias que estavam vigentes ao final de 2017, em suas diferentes fases, na Tabela 1 abaixo, bem como do número de instituições públicas e privadas envolvidas nesses processos. 
Tabela 1 - Parcerias para o Desenvolvimento Produtivo (PDPs) - Posição em 31/12/2017

\begin{tabular}{|c|c|c|c|}
\hline \multicolumn{2}{|c|}{ PDPs em execução } & \multicolumn{2}{|c|}{ Consolidado das PDPs Aprovadas e em Execução } \\
\hline Medicamentos & 67 & Medicamentos & 96 \\
\hline $\begin{array}{l}\text { Equipamentos e materiais e } \\
\text { produtos para diagnósticos }\end{array}$ & 12 & $\begin{array}{l}\text { Equipamentos e materiais e } \\
\text { produtos para diagnósticos }\end{array}$ & 13 \\
\hline Vacinas & 5 & Vacinas & 5 \\
\hline Total Geral & 84 & Total Geral & 114 \\
\hline \multicolumn{2}{|c|}{ PDPs de Pesquisa e Desenvolvimento } & \multicolumn{2}{|c|}{$\begin{array}{c}\text { Produtos em transferência e desenvolvimento de } \\
\text { Tecnologia nas PDPs }\end{array}$} \\
\hline Medicamentos & 4 & Medicamentos & 50 \\
\hline $\begin{array}{l}\text { Equipamentos e materiais e } \\
\text { produtos para diagnósticos }\end{array}$ & 1 & $\begin{array}{l}\text { Equipamentos e materiais e } \\
\text { produtos para diagnósticos }\end{array}$ & 12 \\
\hline Vacinas & 0 & Vacinas & 5 \\
\hline Total Geral & 5 & Total Geral & 67 \\
\hline \multicolumn{2}{|c|}{ Novas PDPs Aprovadas (em fase de formalização) } & \multicolumn{2}{|c|}{$\begin{array}{c}\text { Produtores com Projetos de Parceria Tecnológica nas } \\
\text { PDPs }\end{array}$} \\
\hline Medicamentos & 25 & Instituições Públicas & 18 \\
\hline $\begin{array}{l}\text { Equipamentos e materiais e } \\
\text { produtos para diagnósticos }\end{array}$ & 0 & Empresas Privadas & 42 \\
\hline Vacinas & 0 & Total Geral & 60 \\
\hline Total Geral & 25 & & \\
\hline
\end{tabular}

Fonte: GADELHA; TEMPORÃO, 2018.

A Figura 3, a seguir, demonstra a trajetória das compras centralizadas do MS totais em relação às compras provenientes de PDPs, entre 2011 e 2014. Fica evidente o ganho de espaço das compras provenientes de PDPs no período, que passaram de 20,63\% para 35,42\% das compras centralizadas totais realizadas pelo MS (as quais também apresentaram elevação no período).

Figura 3: Aquisições efetivas de PDP nas compras do MS, 2011- 2014 (em R\$ bilhões)

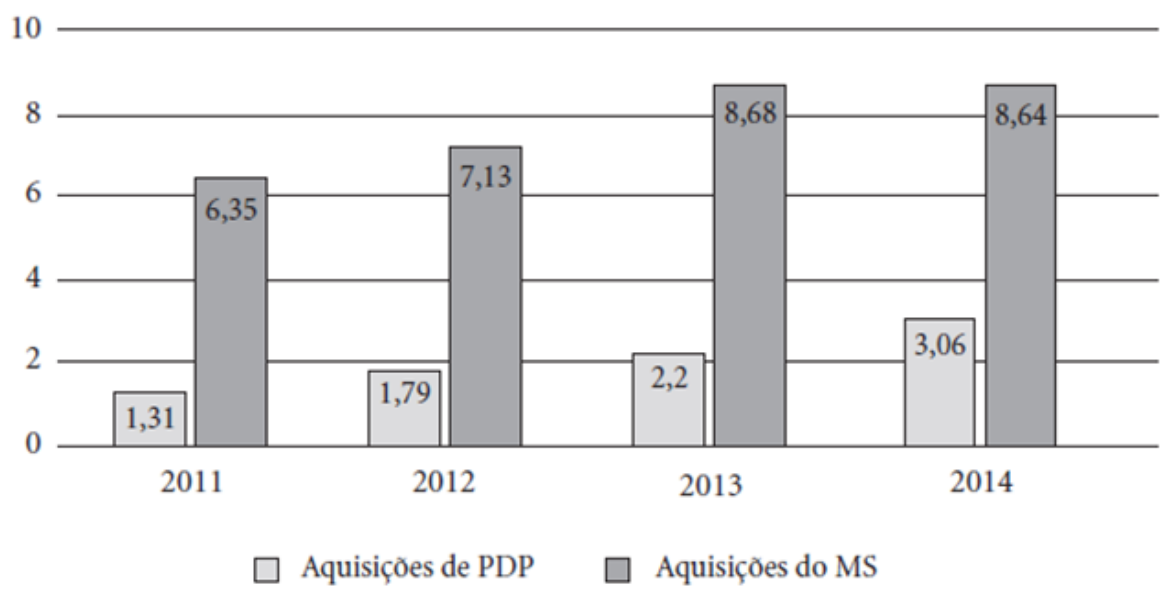

Fonte: GADELHA; TEMPORÃO, 2018, p. 1899.

Gadelha e Temporão (2018) também enfatizam os importantes impactos gerados em termos de elevação da segurança sanitária proporcionados pelas PDPs, uma vez que há capacitação tecnológica das instituições públicas para a produção de tecnologias estratégicas e efeitos importantes sobre as estruturas oligopolísticas já consolidadas, que dificultam tal controle. Por mais que ainda sejam grandes os desafios a serem enfrentados para a capacitação tecnológica adequada dos Laboratórios Farmacêuticos Oficiais (LFOs), de forma que sejam capazes de fazer frente a setores que estão entre os que mais investem em pesquisa e desenvolvimento no mundo, eles ocupam um papel extremamente estratégico no quadro das PDPs, pois permitem uma condução mais efetiva das políticas industriais direcionadas ao setor e também uma maior autonomia para a consecução do objetivo vinculado à elevação do acesso de produtos essenciais à manutenção do SUS (ALMEIDA, 2018). Tais instituições, conforme apresentam Chaves et al. (2018), têm concentrado-se, neste primeiro momento das PDPs, em receber transferências de 
tecnologia de produtos e processos de produtos que já se encontram presentes no mercado nacional, representando, portanto, um baixo grau de inovação, em termos sistêmicos, em um movimento que se direciona mais à capacitação produtiva do que tecnológica. No entanto, apesar de ainda serem grandes os desafios associados à elevação efetiva do potencial inovativo dos LFOs, é inegável que as PDPs proporcionaram a essas instituições uma reinserção na cadeia produtiva farmacêutica nacional, e os avanços associados à capacitação produtiva desses laboratórios, ainda que tímidos, demonstram-se essenciais para o alcance de etapas em que se visione uma capacidade inovativa mais autônoma e elevada (ALMEIDA, 2018).

Além disso, a condução das PDPs, de acordo com Gadelha e Temporão (2018), tem levado a uma tendência de longo prazo para que os preços de aquisição dos produtos objetos de PDPs tenham maior estabilidade, fazendo frente aos movimentos do mercado mundial. Além disso, estimativas realizadas pela SCTIE/MS apresentam que, entre maio de 2011 e maio de 2017, o MS acumulou uma economia de gastos em torno de $\mathrm{R} \$ 4,68$ bilhões (comparando-se os preços do ano anterior às PDPs com os praticados posteriormente à sua implementação). Os autores também alertam quanto estratégias de redução temporária de preços por parte das empresas afetadas negativamente pelas PDPs, utilizadas para manter as suas posições de mercado, enfatizando o caráter de análise estática por parte governamental no caso de aquisições feitas neste contexto, visando a uma busca irrefletida pelo menor preço, em detrimento das PDPs: sem a capacitação dos LFOs, os preços poderão voltar à trajetória de alta, sem que haja elementos que possam fazer contraposições a tal movimento. Enfatizam, assim, a importância da autonomia tecnológica e da visão sistêmica e dinâmica na regência deste processo. Também apresentam como um problema a ser superado o fato de que apenas as compras centralizadas do MS podem ser objetos de PDPs, o que restringe o alcance de tal política.

\section{CONSIDERAÇÕES FINAIS}

Ainda que distante a concretização do sonho de um sistema público de saúde universal, equânime e integral no Brasil, Gadelha (2003), ao introduzir o conceito de Complexo Econômico-Industrial da Saúde, abre novas grandes possibilidades de enfrentamento das dificuldades relacionadas às políticas sociais, de maneira geral, no país, pois parte de um conceito de desenvolvimento amplo, que não considera os desenvolvimentos social e produtivo em esferas isoladas, muito pelo contrário: entende que ambos devem estar profundamente relacionados, devendo constituir partes de políticas integradas e bem coordenadas (GADELHA, 2007).

O presente artigo procurou explorar, dentro dessa perspectiva, as ações que foram tomadas nos últimos anos no sentido de busca de concretização desta ideia. A partir do levantamento bibliográfico apresentado, percebe-se que, dentre os vários esforços foram realizados sob tal inspiração, as Parcerias para o Desenvolvimento Produtivo demonstraram-se como uma alternativa que foi capaz de coordenar os esforços de diversas instituições e de conciliar o uso do amplo poder de compra do Estado como elemento de estímulo à transformação do sistema produtivo de saúde brasileiro, procurando conciliar as perspectivas de ampliação do acesso aos produtos essenciais ao funcionamento do SUS com a diminuição da dependência tecnológica vivenciada pelo parque produtivo nacional. Neste primeiro momento avaliado, como exposto por Chaves et al. (2018), as PDPs exerceram mais um papel de capacitação produtiva do que de capacitação tecnológica, tendo apresentado avanços tímidos sobre as atividade de Pesquisa, Desenvolvimento e Inovação dos LFOs. Como causas possíveis desse cenário, os autores apresentam elementos complicadores, como a ausência de linhas de crédito destinadas à inovação, a excessiva burocracia, a descontinuidade de demanda, as dificuldades de coordenação entre as ações do MS e dos LFOs, entre outros. No entanto, também identificam na própria arquitetura da política dificuldades de criação de ampla capacidade inovativa, uma vez que elas baseiam-se em uma arquitetura que pressupõe a demanda prévia por parte do MS de produtos avaliados como estratégicos ao SUS, em sua maioria, já existentes no mercado. Ou seja: há agregação de produtos novos do ponto de vista dos laboratórios públicos, mas estes não representam inovações diante do mercado, como um todo. No 
entanto, como expõe Almeida (2018), este panorama não pode ser avaliado sem que se leve em conta as dificuldades estruturais associadas às capacidades produtiva e tecnológica destes laboratórios, em que esteve presente um amplo processo de sucateamento histórico de grande parte de suas estruturas produtivas, evidenciando que a ação das PDPs no sentido do reestabelecimento dos LFOs na cadeia farmacêutica nacional possui grandes potencialidades, demonstrando-se como um passo inicial essencial para a consolidação do padrão de desenvolvimento proposto pelo conceito do CEIS.

Mesmo levando-se em consideração as dificuldades apresentadas, diante de um cenário em que os setores produtivos ligados à saúde no Brasil enfrentam dificuldades de ordem estrutural, envolvendo amplas assimetrias em relação aos padrões produtivos e de pesquisa, desenvolvimento e inovação praticados pelas empresas que atuam na fronteira tecnológica mundial, fica evidente que os desafios a que as políticas apresentadas se propõem a enfrentar não são passíveis de serem superados apenas por ações que contemplam o curto prazo, exigindo, como expõe Gadelha (2016), esforços amplos, que envolvam as várias frentes de política econômica e social, e que se baseiem em perspectivas de análise estratégica, levando em conta os efeitos sistêmicos e dinâmicos das ações a serem realizadas.

Por mais que o contexto político e econômico atual tenda a vertentes político-ideológicas que aparentam caminhar em um sentido cada vez mais contrário à concepção mais abrangente de desenvolvimento, colocando em risco as ainda incipientes iniciativas tomadas durante os últimos anos, é importante que se enfatizem, cada vez mais, novos olhares que permitam a sustentabilidade do projeto carregado pelo SUS, o qual, constituído sob um cenário de amplas mobilizações sociais e políticas, propõe-se a enfrentar, de maneira estrutural, as profundas desigualdades em que se pauta a formação histórica da sociedade brasileira, que geram amplos reflexos nas condições em que se concretizam as possibilidades de acesso à saúde dos diferentes grupos sociais. O conceito do CEIS permite, assim, o vislumbre de um projeto nacional que proporciona bases materiais à consolidação do SUS, evidenciando os efeitos econômicos positivos que podem ser atrelados aos gastos públicos em saúde, quando bem direcionados e pensados de forma sistêmica. Como expõem Padula, Noronha e Mitidieri (2016), diante de importantes mudanças paradigmáticas nas tecnologias de produção e de oferta de serviços da indústria de saúde mundial, a ausência de políticas que procurem enfrentar os grandiosos desafios relacionados ao fortalecimento da indústria nacional de saúde, incluindo, neste panorama, a produção pública, colocará o Brasil em uma posição de vulnerabilidade crescente, tornando cada vez mais distantes as possibilidades de garantia do direito constitucional previsto para acesso de toda a população à saúde de forma integral e equânime.

\section{REFERÊNCIAS BIBLIOGRÁFICAS:}

ABRAhÃO, J. MOSTAFA, J.; HERCUlANO, P. Gastos com a Política Social: alavanca para o crescimento com distribuição de renda. Comunicado do IPEA (Instituto de Pesquisas Econômicas Aplicadas), $\quad \mathrm{n}^{0} 75, \quad 2011 . \quad$ Disponível $\quad$ em: http://repositorio.ipea.gov.br/bitstream/11058/4634/1/Comunicados_n75_Gastos_com.pdf $\$$. Acesso em 10/05/2019.

ALMEIDA, A. C. S. Análise dos efeitos das Parcerias para o Desenvolvimento Produtivo nos Laboratórios Farmacêuticos Oficiais. Tese de Doutorado apresentada ao Programa de Pós-graduação em Economia do Instituto de Economia da Universidade Federal do Rio de Janeiro. Rio de Janeiro, 2018.

BNDES - Banco Nacional de Desenvolvimento. Relatório Anual - 2011. Rio de Janeiro, 2011. Disponível em:

https://www.bndes.gov.br/SiteBNDES/export/sites/default/bndes_pt/Galerias/Arquivos/empresa/RelAnua 1/ra2011/relatorio_anual2011.pdf $>$. Acesso em 04/04/2019. 
BRASIL. Ministério da Saúde. Secretaria de Políticas de Saúde. Departamento de Atenção Básica. Política Nacional de Medicamentos. Ministério da Saúde, Secretaria de Políticas de Saúde, Departamento de Atenção Básica. Brasília, 2001.

BRASIL. Decreto n ${ }^{\circ} 7.713$, de 3 de abril de 2012a.

BRASIL. Decreto n ${ }^{\circ} 7.767$, de 27 de junho de 2012 b.

BRASIL. Portaria ${ }^{\circ}$ 2.531, de 12 de novembro de 2014.

BRASIL. Decreto n ${ }^{\circ}$ 9.245, de 20 de dezembro de 2017.

BRASIL. Ministério da Saúde. Saúde de A a Z: Parcerias para o Desenvolvimento Produtivo (PDP). Publicado em 29 de janeiro de 2019. Disponível em: <http://portalms.saude.gov.br/saude-de-az/parcerias-para-o-desenvolvimento-produtivo-pdp>. Acesso em 15/05/2019.

CALDEIRA, T. R. Acesso ao medicamento: direito à saúde no marco da regulação do mercado farmacêutico. 2010. Dissertação (Mestrado em Política Social) - Departamento de Serviço Social, Instituto de Ciências Humanas Universidade de Brasília, Brasília, 2010.

CANO, W.; SILVA, A. L. G. A política industrial do Governo Lula. Texto para Discussão, IE-Unicamp, n.181, 27p. 2010.

CAPANEMA, L. X. L. A indústria farmacêutica brasileira e a atuação do BNDES. BNDES Setorial, n. 23, p. 193-216, Rio de Janeiro, 2006.

CHAVES, G. C.; AZEREDO, T. B.; VANSCONCELOS, D. M. M.; MENDOZA-RUIZ, A.; SCOPEL, C. T.; OLIVEIRA, M. A.; HASENCLEVER, L. Produção pública de medicamentos no Brasil: capacitação tecnológica e acesso. $1^{\text {a }}$ ed. Rio de Janeiro: E-Papers, 2018.

CONSELHO FEDERAL DE MEDICINA. Demografia médica 2015: população que depende do SUS tem três vezes menos médicos que usuários de planos de saúde. CFM, 2015. Disponível em $<$ https://portal.cfm.org.br/index.php?option=com_content\&view=article\&id=25875>. Acesso em 07/05/2019.

FAGNANI, E. Política Social no Brasil (1964-2002): entre a cidadania e a caridade. Tese de Doutorado em Ciências Econômicas. Instituto de Economia da UNICAMP, Campinas, 2005.

GADELHA, C. A. G. O Complexo Industrial da Saúde e a necessidade de um enfoque dinâmico na economia da saúde. Ciência \& Saúde Coletiva, v. 8, n.2, p. 521-535, Rio de Janeiro, 2003.

GADELHA, C. A. G. Desenvolvimento e Saúde: em busca de uma nova utopia. Saúde em Debate; v. 19, n. 71, p. 326-327, set/dez, Rio de Janeiro, 2007.

GADElHA, C. A. G.; BARBOSA, P. R.; MALDONADO, J.; VARGAS, M. A.; COSTA, L. O Complexo Econômico-Industrial da Saúde: conceitos e características gerais. VPPIS/FIOCRUZ - Informe CEIS n ${ }^{\mathbf{0}}$ 1, ano 1, Rio de Janeiro, 2010.

GADELHA, C. A. G.; COSTA, L. S.; MALDONADO, J. O Complexo Econômico-Industrial da Saúde e a dimensão social e econômica do desenvolvimento. Revista Saúde Pública, v.46, p.21-28, 2012. 
GADELHA, C. A. G.; COSTA, L. S. A saúde na política nacional de desenvolvimento: um novo olhar sobre os desafios da saúde. In: FUNDAÇÃO OSWALDO CRUZ. A saúde no Brasil em 2030 Prospecção estratégica do sistema de saúde brasileiro - Desenvolvimento, Estado e políticas de saúde. V. 1, p. 101 - 132. Rio de Janeiro, 2013.

GADELHA, C. A. G.; BRAGA, P. S. C. Saúde e inovação: dinâmica econômica e Estado de Bem-Estar Social no Brasil. Cad. Saúde Pública, 32, Sup. 2, Rio de Janeiro, 2016.

GADELHA, C. A. G. Política industrial, desenvolvimento e os grandes desafios nacionais. In: SARTI, F.; LAPLANE, G.; CASSIOLATO, J. E.; LASTRES, H. M. M. O futuro do desenvolvimento. P. 215-251. Instituto de Economia da UNICAMP, Campinas, 2016.

GADELHA, C. A. G. A saúde é a alavanca para sair da crise. Entrevista concedida à Região e Redes: caminhos da universalização da saúde no Brasil, publicada em 13 de setembro de 2017. Disponível em: $<$ http://www.resbr.net.br/a-saude-e-alavanca-para-sair-da-

crise/?fbclid=IwAR0ye50YDhqHuJX51w8AfpW1gQCcjfk5MUlaYqWly0BSquUokvxdhlSpYnA\#.XA7 58GhKjIV>. Acesso em: 05/05/2019.

GADELHA, C. A. G.; TEMPORÃO, J. G. Desenvolvimento, Inovação e Saúde: a perspectiva teórica e política do Complexo Econômico-Industrial da Saúde. Ciência e Saúde Coletiva, v. 23, n.6, p. 1891-1902, Rio de Janeiro, 2018.

GOMES, R.; PIMENTEL, V.; LOUSADA, M.; PIERONI, J. P. O novo cenário de concorrência na indústria farmacêutica brasileira. BNDES Setorial, n. 39, p. 97-134, BNDES, Rio de Janeiro, 2013.

NOVAIS, L. F.; QUINTÃO, M. A.; CAGNIN, R. F. Panorama Mundial e Brasileiro do Setor Farmacêutico. Instituto de Estudos para o Desenvolvimento Industrial, dez. de 2016.

OCKÉ-REIS, C. O.; FUNCIA, F. R. Terror Fiscal e Desmonte do SUS: uma ameaça à democratização da saúde. Plataforma Política Social. 2016. Disponível em: <http://plataformapoliticasocial.com.br/artigo40-terror-fiscal-e-desmonte-do-sus-uma-ameaca-a-democratizacao-da-saude/\#_ftnrefl>. Acesso em: $02 / 05 / 2019$.

OLIVEIRA, G. R. R. Desenvolvimento Econômico e Social: o caso do Complexo Econômico Industrial de Saúde. Trabalho de Conclusão de Curso de Graduação em Ciências Econômicas. Instituto de Economia - UNICAMP. Campinas, 2017.

ORAIR, R. O.; SIQUEIRA, F. F.; GOBETTI, S. W. Política Fiscal e Ciclo Econômico: uma análise baseada em multiplicadores do gasto público. Monografia premiada em 2o lugar. XXI Prêmio Tesouro Nacional. Brasília, 2016.

PADULA, R.; NORONHA, G. S.; MITIDIERI, T. L. Complexo Econômico-Industrial da Saúde, segurança e autonomia estratégica: a inserção do Brasil no mundo, In: GADELHA, C. A. G.; GADELHA, P.; NORONHA, J. C. (orgs.) Brasil Saúde Amanhã: Complexo Econômico-Industrial da Saúde. P. 173-227. Rio de Janeiro: Editora FIOCRUZ, 2016.

SALDIVA, P. H. N.; VERAS, M. Gastos públicos com saúde: breve histórico, situação atual e perspectivas futuras. Estudos Avançados, v. 32, n.92, p. 47-61, 2018

SILVA, E. N. Complexo Econômico-Industrial da Saúde. Tempus Actas de Saúde Coletiva, v. 8, n. 1, p. 57-62, Brasília, 2014. 